\title{
EVALUACIÓN DE LA FUNCIÓN VENTRICULAR IZQUIERDA MEDIANTE ECOCARDIOGRAFÍA CON SPECKLE TRACKING LUEGO DEL CIERRE PERCUTÁNEO DEL CONDUCTO ARTERIOSO PERMEABLE. EXPERIENCIA DEL HOSPITAL DE NIÑOS “ROBERTO GILBERT ELLZALDE”
}

\author{
EVALUATION OF THE LEFT VENTRICULAR FUNCTION THROUGH SPECKLE TRACKING \\ ECOCARDIOGRAPHY AFTER THE PERCUTANEOUS CLOSURE OF THE PERMEABLE ARTERIOUS DUCT. \\ EXPERIENCE OF THECHILDREN'S HOSPITAL "ROBERTO GILBERTELIZALDE"
}

\begin{abstract}
AVALIAÇÃO DA FUNÇ̄ÃO VENTRICULAR ESQUERDA ATRAVÉS ECOCARDIOGRAFIA COM RASTREAMENTO DESPECKLE APÓS O ENCERRAMENTO PERCUTÂNEO DO DUTO ARTERIOSO PERMEÁVEL. EXPERIÊNCIA DO HOSPITAL DE CRIANÇAS

"ROBERTO GILBERT ELLZALDE"
\end{abstract}

\section{MARCO DURÁN ALEMÁN', PAOLA MENDIETA CHISPE', BORIS BARRENO MARTÍNEZ', SIMÓN DUQUE SOLÓRZANO'}

${ }^{1}$ Hospital Roberto Gilbert Elizalde, Guayaquil, Ecuador.

Resumen

Objetivo: evaluar la función del ventrículo izquierdo (VI) mediante métodos convencionales de ecocardiografía y de deformación miocárdica con speckle tracking antes y después del cierre percutáneo del conducto arterioso permeable (CAP). Materiales y métodos: la población estudiada comprendió 20 niños con CAP hemodinamicamente significativo en los que se practicó el cierre percutáneo. Mediante ecocardiografía-Doppler y speckle tracking se valoró el tamaño ventricular izquierdo, fracción de eyección y la deformidad miocárdica antes y después del procedimiento. Resultados: el diámetro del VI en diástole (DVID) disminuyó de $44,78 \mathrm{~mm}( \pm 11,9)$ a 41,28 mm ( $\pm 8,7)$ luego del cierre $(\mathrm{p}=0.028)$. El promedio de la fracción de eyección FE $64,17 \%( \pm 5,7)$ y la Fracción de Acortamiento (FAc) 36\% $( \pm 5,6)$ cayeron a $60,3 \%( \pm 9,3)$ y a $32,8( \pm 6,0)$ respectivamente, sin alcanzar valor estadístico. De acuerdo a los criterios predefinidos se registraron 5 pacientes $(25 \%)$ con disfunción sistólica ( $\mathrm{FE}=<50 \%$ ) luego del procedimiento, su $\mathrm{FE}$ al inicio fue $66 \%( \pm 7,31)$ cayendo a $47 \%$ ( \pm 3$)$ luego del cateterismo $(\mathrm{p}<0.05)$. Estudiando la deformación miocárdica, antes del cierre el promedio del strain longitudinal fue $-23,41 \%( \pm 2,32)$ para, luego del procedimiento descender a $-20,12 \%( \pm 3,38)$ $(p<0,01)$. Conclusiones: el cierre percutáneo del CAP provoca una temprana disminución en la función miocárdica del VI que se recupera a las pocas semanas. El estudio ecocardiográfico convencional más el strain miocárdico podría ser un fuerte predictor de disfunción ventricular luego del cateterismo.

PALABRAS CLAVE: conducto arterioso permeable, cateterización cardíaca, ecocardiograma, Spekle Tracking.

Abstract

Objective: to evaluate the function of the left ventricle (LV) by conventional methods of echocardiography, and the myocardial deformation with speckle tracking before and after the percutaneous closure of patent ductus arteriosus (PDA). Materials and methods: the population studied comprised 20 children with hemodynamically significant PDA in whom percutaneous closure was practiced. Using Doppler echocardiography and speckle tracking, we assessed the left ventricular size, ejection fraction and myocardial deformity before and after the procedure. Results: the diameter of the LV in diastole (DLVD) decreased from 44.78 $\mathrm{mm}( \pm 11.9)$ to $41.28 \mathrm{~mm}( \pm 8.7)$ after closure $(p=0.028)$. The mean ejection fraction EF $64.17 \%( \pm 5.7)$ and the Shortening Fraction $(\mathrm{ShF}) 36 \%( \pm 5.6)$ fell to $60.3 \%$ $( \pm 9.3)$ and to 32.8. $( \pm 6.0)$ respectively, without reaching statistical value. According to the predefined criteria, 5 patients $(25 \%)$ with systolic dysfunction $(E F=<50 \%)$ were registered after the procedure, their $E F$ at the beginning was $66 \%( \pm 7.31)$, falling to $47 \%( \pm 3)$ after catheterization $(p<0.05)$. When studying the myocardial deformation, before the closure, the average longitudinal strain was $-23.41 \%( \pm 2.32)$, after the procedure, it decreased to $-20.12 \%( \pm 3.38)(p<0.01)$. Conclusions: the percutaneous closure of PDA causes an early decrease in LV myocardial function that recovers after a few weeks. The conventional echocardiographic study plus myocardial strain could be a strong predictor of ventricular dysfunction after catheterization

KEYWORDS: patent ductus arteriosus, cardiac catheterization, echocardiogram, spekle tracking.

Resumo

Objetivo: Avaliar a função do ventrículo esquerdo (VE) pelos métodos convencionais de ecocardiografia e deformação miocárdica com speckle tracking antes e após o fechamento percutâneo da persistência do ducto arterioso (CAP). Materiais e métodos: a população estudada foi composta por 20 crianças com PAC hemodinamicamente significativa, nas quais o fechamento percutâneo era praticado. Usando ecocardiograma-Doppler e rastreamento de speckle avaliaram 0 tamanho do ventrículo esquerdo, a fração de ejeção e a deformidade miocárdica antes e após o procedimento. Resultados: 0 diâmetro do VE em diástole (DVID) diminuiu de $44,78 \mathrm{~mm}( \pm 11,9)$ para $41,28 \mathrm{~mm}( \pm 8,7)$ após o fechamento $(\mathrm{p}=0,028)$. A fração de ejeção média $F E 64,17 \%$ ( $\pm 5,7)$ e a fração de encurtamento (FAc) $36 \%( \pm 5,6)$ caíram para $60,3 \%( \pm 9,3)$ e para 32,8 . $( \pm 6,0)$, respectivamente, sem atingir valor estatístico. De acordo com os critérios pré-definidos, 5 pacientes (25\%) com disfunção sistólica ( $\mathrm{FE}=<50 \%$ ) foram registrados após o procedimento, sua $\mathrm{FE}$ no início foi de $66 \%( \pm 7,31)$, caindo para $47 \%( \pm 3)$ depois de cateterismo $(p<0,05)$. Ao estudar a deformação miocárdica, antes do fechamento, a deformação longitudinal média foi de $-23,41 \%( \pm 2,32)$, pois, após o procedimento, diminuiu para $-20,12 \%( \pm 3,38)(p<0,01)$. Conclusões: o fechamento percutâneo do CAP provoca uma diminuição precoce da função miocárdica do VE que se recupera após algumas semanas. 0 estudo ecocardiográfico convencional mais a tensão miocárdica poderia ser um forte preditor de disfunção ventricular após o cateterismo.

PALAVRAS-CHAVE: persistência do canal arterial, cardíaco cateterismo, ecocardiografía, speckle tracking. 
INTRODUCCIÓN

El conducto arterioso permeable (CAP) es una estructura vascular con un papel esencial en la circulación fetal que luego de pocas semanas del nacimiento debe ocluirse espontáneamente. De mantenerse su permeabilidad, el conducto arterioso se constituye en una de las cardiopatías congénitas más frecuentes. ${ }^{1} \mathrm{El} \mathrm{CAP}$ provoca un cortocircuito de izquierda a derecha con sobrecarga de volumen y el consiguiente remodelado ventricular izquierdo (VI), cambios cuya magnitud depende entre otras causas, de las características anatómicas del propio conducto, de las presiones derechas, alteraciones que son fácilmente detectadas y cuantificadas mediante ecocardiografía. ${ }^{2}$

El tratamiento del CAP puede ser quirúrgico o por vía percutánea, técnica esta última que ha demostrado ser efectiva y segura ${ }^{3}$ con resultados comparables a los alcanzados mediante cirugía por lo que es actualmente considerada como de primera elección. En muchos casos, la súbita eliminación de la sobrecarga de volumen que ocurre inmediatamente después del cierre, provoca una abrupta disminución de la elongación de la fibra miocárdica que podría conducir a disfunción ventricular muchas veces sintomática. ${ }^{4,5}$

Como propósito de este estudio se plantea investigar mediante ecocardiografía convencional y speckle tracking los cambios hemodinámicos que ocurren en el VI antes y luego del cierre percutáneo del CAP así como intentar identificar sus factores asociados

\section{MATERIALES Y MÉTODOS}

Entre diciembre de 2014 y mayo de 2016 fueron seleccionados los pacientes que con el diagnóstico clínico de CAP acudieron al laboratorio de ecocardiografía donde se confirmó la presencia de dicha malformación hemodinamicamente significativa y que posteriormente fueron programadas para cierre percutáneo. De estos pacientes fueron excluidos aquellos que presentaron defecto cardiaco asociado, estudio ecocardiográfico incompleto (sin análisis de la deformidad miocárdica), calidad inadecuada del estudio, procedimiento percutáneo fallido y pacientes quienes fueron sometidos a intervenciones concomitantes al cierre de CAP.

ECOCARDIOGRAFIA BIDIMENSIONAL Y SPECKLE TRACKING Los estudios ecocardiográficos fueron obtenidos con un equipo Vivid 9 General Electric (CE Medi- cal Systems Milwaukee, USA) utilizando sondas de 3.5 MHz (M5S) ó 6.0 MHz (6S) dependiendo de la edad del niño. El estudio previo fue obtenido el día anterior al procedimiento y el estudio de control se realizó entre 24 y 72 horas luego del cateterismo. Las dimensiones, espesor de paredes, Fracción de Eyección (FE) y Fracción de Acortamiento (FAc) del ventrículo izquierdo se determinaron mediante ecocardiografía modo $M$ derivada de las imágenes 2D desde el eje largo vista paresternal. Se consideró como disfunción sistólica una $\mathrm{FE}=<50 \%$ postoclusión.

Los estudios con speckle-tracking se realizaron exclusivamente desde el plano apical (2, 3 y 4 cámaras) siguiendo el protocolo del laboratorio á disposición. ${ }^{6}$

CATETERISMO CARDIACO

Después de obtener el consentimiento informado se realizó el cateterismo cardiaco obteniéndose aortografías en proyecciones anterior oblicua derecha $40^{\circ}$ y lateral para definir posición, forma y dimensiones del CAP. El tamaño del dispositivo se escogió añadiendo $2 \mathrm{~mm}$ al diámetro menor del conducto arterioso.

El procedimiento para el cierre se realizó bajo anestesia general. Por vía femoral se obtuvo acceso arterial y venoso, se midió saturación y presiones en arco aórtico y arteria pulmonar antes del cierre. Luego de desplegar dispositivo se realizaron controles angiográficos desde la aorta para comprobar la posición adecuada del dispositivo, cierre completo de CAP y la exclusión de fugas residuales significativas. Después de comprobar la ausencia de obstrucciones en extremos aórtico y pulmonar, se retiran los accesos vasculares y se deja apósito compresivo en sitios de punción.

Todos los pacientes recibieron profilaxis con antibióticos (cefazolina) y heparina $50 \mathrm{ui} / \mathrm{kg}$ durante el procedimiento

\section{ANÁLISIS ESTADÍSTICO}

El análisis estadístico fue desarrollado usando minitab 17 software.

Las variables categóricas se expresaron como frecuencia absoluta y porcentajes y fueron comparadas usando el test exacto de Fisher.Las variables continuas se expresaron como media \pm desviación estándar para luego ser comparadas usando el test de t student. 
Se dicotomizó la variable fracción de eyección en pre y post cierre del conducto arterioso y se usó diferencia de medias pareadas para determinar diferencia significativa entre los mismos. Una p $<0.05$ se consideró de significancia estadística con un nivel de confianza del 95\%.

\section{RESULTADOS}

En el presente estudio se enrolaron 32 pacientes de los cuales, por razones mencionadas fueron excluidos 12 niños quedando para el análisis 20 pacientes. Las características antropométricas se resumen en la tabla 1 . El promedio de edad fue 8,75 años $( \pm 6,33)$ rango 7 meses a 18 años, de sexo femenino fueron $17(84,2 \%)$ El peso promedio fue de $28,58( \pm 20,15) \mathrm{k}$ con un rango de 7 a 80,8 . El promedio de la estatura fue $116,8 \mathrm{~cm}( \pm 33,5)$ rango 71 - $162 \mathrm{~cm}$ y la superficie corporal promedio 0,96 $\mathrm{m} 2( \pm 0,46)$ con rango de $0,37-1,91 \mathrm{~m} 2$.

TABLA 1. CARACTERÍSTICAS DEMOGRÁFICAS DE LOS PACIENTES PDA

\begin{tabular}{lrrr} 
& PROMEDIO & $\mathbf{\pm D E}$ & RANGO \\
\hline Edad (años) & 8,75 & 6,33 & $7 \mathrm{~m}-18 \mathrm{a}$ \\
\hline Peso (K) & 28,58 & 20,15 & $7-80,8$ \\
\hline Talla (cm) & 116,8 & 33,5 & $71-162$ \\
\hline S.C.m2 & 0,96 & 0,46 & $0,37-1,91$ \\
\hline
\end{tabular}

años (a), kilos (k), metros cuadrados (m2), superficie corporal (S.C.).

ECOCARDIOGRAFÍA

Comparando los valores ecocardiográficos basales con los obtenidos luego del cierre percutáneo (tabla 2) se observaron algunas diferencias. El valor promedio del diámetro del VI en diástole (DVID) antes del cierre fue $45,8 \mathrm{~mm}( \pm 11,7)$ para, luego del procedimiento cambiar a 41,3 $\mathrm{mm}( \pm 8,7)$ con valor estadístico significativo (p $<0.05$ ) El diámetro sistólico (DSVI) disminuyó de $29,20 \mathrm{~mm}( \pm 7,68)$ basal a $28,40 \mathrm{~mm}( \pm 7,40)$ luego del procedimiento, cambio que no alcanzó significación estadística. En condiciones basales ninguno de los pacientes presentó disfunción sistólica del VI siendo el valor promedio de la FE $64,17( \pm 5,7)$ y la FAc $36 \%( \pm 5,6)$. Luego del procedimiento se observó una evidente disminución a $60,3 \%( \pm 9,3)$ y a $32,8( \pm 6,0)$ respectivamente, sin embargo estos cambios no alcanzaron valor estadístico (p 0.19). De acuerdo a los criterios predefinidos se registraron 5 pacientes $(25 \%)$ con disfunción sistólica $(\mathrm{FE}=<50 \%)$ luego del procedimiento. Dentro de este subgrupo la FE al inicio fue $66 \%( \pm 7,31)$ cayendo a $47 \%( \pm 3)$ post cateterismo con valor estadísticamente significativo $(\mathrm{p}<0,01)$ En casi todos los pacientes la FE y la FAc volvieron a valores normales a las 48 horas de la intervención en forma espontánea.
TABLA 2. VALORES ECOCARDIOGRÁFICOS ANTES Y DESPUÉS DEL CIERRE PDA

\begin{tabular}{lrrr}
\hline & PRE CIERRE PERCUTÁNE0 & POST & P $<0,01$ \\
\hline DVLd & $44,78 \pm 11,94$ & $41,28 \pm 8,7$ & 0.02 \\
\hline DVIs & $29,20 \pm 7,68$ & $28,40 \pm 7,68$ & NS \\
\hline SIV & $6,82 \pm 1,55$ & $6,64 \pm 1,11$ & NS \\
\hline PPVI & $6,61 \pm 1,72$ & $60,33 \pm 9,8$ & NS \\
\hline FE & $64,75 \pm 9,39$ & $32,8 \pm 6,0$ & $\mathrm{NS}$ \\
\hline FAc & $35,95 \pm 5,8$ & & \\
\hline FE $=<50 \%$ POST CIERRE & & & \\
\hline FE & $66 \% \pm 7,31$ & $47 \% \pm 3$ & $\mathrm{p}<0,01$ \\
\hline
\end{tabular}

DVId: diametro ventricular izquierdo en diástole, DVls: diámetro ventricular en sístole, FE: fracción de eyección, FAc: fracción de acortamiento, ns: no significativo, PPVI: pared posterior de ventrículo izquierdo, SIV: septo interventricular.

En el grupo de pacientes con disfunción sistólica su recuperación fue más lenta, recibieron el alta un promedio de 72 horas y en un caso de este grupo que presentó clínica de fallo ventricular, hubo necesidad de soporte farmacológico. Al comparar el diámetro pulmonar (AP) del CAP con la FE, no se encontró significación estadística. Observando que el diámetro pulmonar del CAP en la cohorte presenta valores muy diversos se decidió (tabla 3) estandarizarlos para la superficie corporal (SC) y comparar nuevamente el cociente (CAP-AP/SC) con la FE. La relación establecida fue inversa, los cocientes más altos presentaron una FE menor aunque con débil significado estadístico $(\mathrm{p}=0.03)$

TABLA 3. VALORES HEMODINÁMICOS DE LOS PACIENTES PDA

\begin{tabular}{|c|c|c|c|c|c|}
\hline & $\begin{array}{l}\text { DIÁMETRO } \\
\text { PULMONAR }\end{array}$ & $\begin{array}{r}\text { SUPERFICIE } \\
\text { CORPORAL }\end{array}$ & $\begin{array}{r}\text { DP / } \\
\text { SC }\end{array}$ & $\begin{array}{r}\text { TIPO DE } \\
\text { DISPOSITIVO }\end{array}$ & $\begin{array}{r}\text { SHUNT } \\
\text { RESIDUAL }\end{array}$ \\
\hline 1 & 9 & 0.42 & 21.43 & VP & NO \\
\hline 2 & 4 & 0.43 & 9.30 & $\mathrm{ADO}$ & NO \\
\hline 3 & 3,5 & 1.16 & 3.01 & ADO & NO \\
\hline 4 & 9 & 0.37 & 24.32 & $\mathrm{ADO}$ & $\mathrm{L}$ \\
\hline 5 & 4,5 & 0.37 & 12.16 & ADO & $\mathrm{L}$ \\
\hline 6 & 4,1 & 1.27 & 3.23 & ADO & NO \\
\hline 7 & 4 & 1.56 & 2,56 & VP & NO \\
\hline 8 & 6 & 1.54 & 3,89 & ADO & NO \\
\hline 9 & 4 & 0.72 & 5,56 & ADO & NO \\
\hline 10 & 3 & 1.91 & 1,57 & ADO & NO \\
\hline 11 & 3 & 0.75 & 4 & $\mathrm{ADO}$ & NO \\
\hline 12 & 4,5 & 1.27 & 3,54 & $\mathrm{ADO}$ & NO \\
\hline 13 & 3,5 & 1.16 & 3,02 & ADO & NO \\
\hline 14 & 3,5 & 0.54 & 6,48 & VP & NO \\
\hline 15 & 3 & 0.49 & 6,12 & ADO & NO \\
\hline 16 & 8 & 1.36 & 5,88 & $\mathrm{ADO}$ & NO \\
\hline 17 & 4,2 & 0.98 & 4,28 & ADO & NO \\
\hline 18 & 3 & 0.89 & 3,37 & ADO & NO \\
\hline 19 & 3,9 & 0.69 & 5,65 & ADO & NO \\
\hline 20 & 5 & 1.34 & 3,73 & ADO & No \\
\hline Promedio & 4,65 & 0.96 & 6.66 & & \\
\hline $\mathrm{DE}$ & \pm 1.90 & \pm 0.46 & \pm 6.07 & & \\
\hline Rango & $3-9$ & $\begin{array}{r}0.37- \\
1.91\end{array}$ & $\begin{array}{r}1.57- \\
24.3\end{array}$ & & \\
\hline
\end{tabular}

ADO Amplatzer Duct Occluder, DP/SC: relación del diámetro pulmonar con la superficie corporal, VP Vascular Plug 
DEFORMIDAD MIOCÁRDICA (SPECKLE TRACKING)

A todos los niños que conforman el estudio se les realizó exploración de la deformación miocárdica mediante speckle tracking antes y después del cateterismo. El valor promedio del strain longitudinal global (SLG) antes de la intervención fue $-23,41 \%( \pm 2,32)$ para luego de la intervención disminuir a $-20,12 \%( \pm 3,38)$ con valor estadísticamente significativo $(\mathrm{p}<0,01)$, (Figura 1).

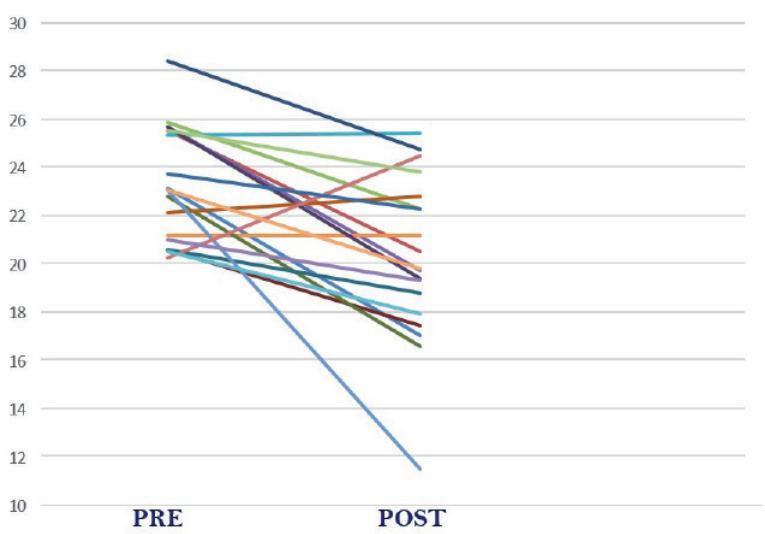

Figura 1. Comparación del strain longitudinal global antes y después del cierre del PDA

Estos cambios, al ser analizados por grupos de paredes evidenciaron una fuerte disminución del porcentaje de deformación en todos ellos, siendo particularmente manifiesto el observado en la paredes ventriculares ántero-septal e inferior (3C), (Figura 2) cambios que lograron valores estadísticamente significativos. 3c

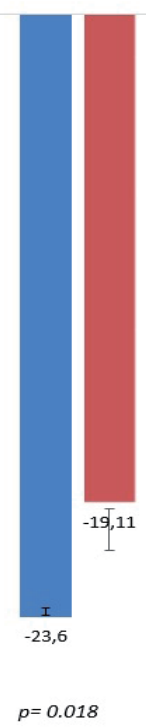

$p=0.004$

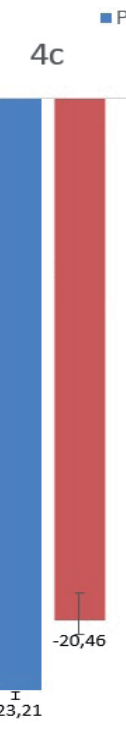

2c

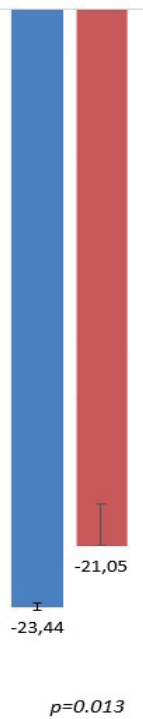

Global

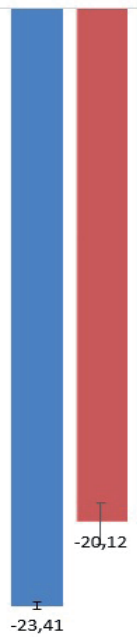

$p=0.0$
Figura 2 Comparación de la deformidad miocárdica antes y después del cierre percutáneo del PDA. Strain longitudinal global y por segmentos. 2C: 2 cámaras, 3C: 3 cámaras, 4 C: 4 cámaras
Comparando los valores del strain longitudinal global con la FE postcateterismo, se obtuvo una relación lineal aunque estadísticamente poco significativa.

\section{HEMODINAMIA}

Mediante cateterismo cardiaco (Tabla 3) se determinó el promedio del diámetro del extremo pulmonar del CAP en 4,65 mm ( \pm 1.90$)$ rango 3 a $9 \mathrm{~mm}$. Se utilizaron dispositivos Amplatzer Ductal Occluder en 17 pacientes (ADO Saint Jude Medical ) y los 3 restantes fueron ocluidos con dispositivos Vascular Plug (Saint Jude Medical). Se observó corto circuito residual en 2 pacientes; en ambos casos fue ligero; hipertension pulmonar en un solo caso.

Se registró una complicación importante producida por migración del dispositivo hacia la arteria pulmonar izquierda. Luego del retiro del dispositivo desplazado se procedió a la colocación de uno nuevo en forma exitosa, sin registrarse flujo residual. El control ecocardiográfico realizado 24 horas después reportó ubicación correcta del dispositivo con oclusión completa del conducto arterioso. Se observó además una importante disminución en la contractilidad miocárdica y manifestaciones clínicas de disfunción ventricular por lo que su alta debió posponerse hasta mejorar su función sistólica que se logró 72 horas después mediante intervención farmacológica

DISCUSIÓN

La técnica percutánea en el cierre del CAP ha tenido una permanente evolución tanto en su eficacia como en su seguridad, por lo que en la actualidad se considera el método de primera elección en el tratamiento de esta cardiopatía congénita. A pesar de ello, se sabe también que después del cierre mediante cateterismo $0^{4,5,7-9}$ es muy frecuente observar en forma temprana alteraciones de la función sistólica ventricular izquierda.

En el presente estudio se comprobó que este efecto adverso se produjo en la mayoría de los pacientes y que la FE descendió $\mathrm{a}=<50 \%$ en 5 niños ( $25 \%$ ) y ocurrió en fase temprana luego del cierre percutáneo. El CAP provoca una sobrecarga de volumen con un impacto hemodinámico sobre el VI determinado, entre otras causas por el diámetro del extremo pulmonar del conducto arterioso. Este exceso de volumen genera a su vez mayor estiramiento de los haces musculares ventriculares estimulando una mayor respuesta 
contráctil con aumento del gasto cardiaco $^{10}$ mediado por la ley de Frank-Starling como se evidencia por el aumento del diámetro diastólico y por el incremento de la deformidad miocárdica (strain) global del VI

Luego de la oclusión del ductus, el corto-circuito de I-D desaparece produciéndose una inmediata reducción de la precarga. El exceso de volumen sanguíneo que antes del cierre se conducía hacia la circulación pulmonar (sistema de menor resistencia), ha sufrido un redireccionamiento luego del cierre, de forma que ahora el volumen sanguíneo se orienta hacia un circuito de mayor resistencia (sistémico), provocando incremento de la poscarga. La disminución del volumen ventricular izquierdo (caída de la precarga) y el aumento de las resistencias sistémicas (aumento de la postcarga), generan un desbalance hemodinámico (afterload mismatch) ${ }^{11}$ que contribuye a la disfunción ventricular.

La disminución de la FE y FAc a valores a veces inferiores a los normales, es un efecto adverso frecuente luego del cierre del CAP ya sea vía percutánea o quirúrgica. En el presente estudio, este fenómeno fue registrado en $5 \operatorname{casos}(25 \%)$ sin que se observaran manifestaciones clínicas de disfunción ventricular excepto en un paciente que requirió intervención farmacológica. Este niño presentó un diámetro ductal pulmonar absoluto y también relativo a la SC, de los más altos, factor que según algunos estudios, ${ }^{8,9,11}$ se considera predictivo de mayor deterioro de la función sistólica luego del procedimiento; sin embargo hay que recordar que en el caso que nos ocupa al ser sometido a una intervención mas prolongada, hubo mayor radiación, mayor sedación, factores que pudieron contribuir al deterioro de la función miocárdica. El tiempo en el que la FE vuelve a la normalidad se muestra variable, podrían ser algunas semanas hasta algunos meses.

Aunque dentro de los objetivos planteados en el presente trabajo no fue el seguimiento a mediano o largo plazo, por las características clínicas de los 5 niños con $\mathrm{FE}=<50 \%$ luego del cierre del CAP, se pudo seguir de cerca su evolución y se observó que la FE volvió a valores normales entre 4- 6 semanas de la intervención, siendo mayor el periodo en el niño que fue sometido a una doble intervención por desplazamiento del dispositivo

A este respecto se han publicado estudios en los que se encontró que la recuperación de la $\mathrm{FE}$ a valores normales, luego del cierre del ductus en adultos, difiere de la de los niños. El tiempo para la normalización de la función sistólica en los niños es mucho menor que en los adultos debido a que en estos, la prolongada sobrecarga de volumen llegaría a generar algún grado de lesión miocárdica que retarde su recuperación. $., 8,11$

Parámetros de deformación miocárdica mediante speckle tracking como el Strain Global, han demostrado tener alta sensibilidad a las modificaciones de pre y poscarga. ${ }^{12}$ En el presente estudio no se observaron cambios estadísticamente significativos en el promedio de la FE ni FAc posoclusión sin embargo tanto el strain miocárdico global como en el de los diferentes segmentos luego del procedimiento presentaron diferencias significativas. Estos cambios traducen grados de deterioro en el rendimiento miocárdico como lo confirman estudios de Kufashi et al ${ }^{13}$ y de Agha et $\mathrm{al}^{14}$. En este último la función miocárdica fue valorada mediante el índice de Tei y luego de compararla con la deformidad miocárdica, encontraron que el Tei se mostraba más prolongado en los pacientes que presentaron valores más altos de strain global antes del cierre del CAP para, luego de 48 horas del procedimiento, tener un descenso significativo, lo cual sugiere que la disfunción sistólica pasajera sea debida a una aguda disminución en la longitud de la fibra miocárdica que se produce por el súbita disminución del volumen ventricular izquierdo. Por su parte, Chonimy et a ${ }^{15}$ utilizan el strain y strain rate y los comparan con la función miocárdica medida también por el índice de Tei posoclusión llegando a conclusiones muy similares.

Siguiendo este razonamiento parece lógico suponer que la deformidad miocárdica en sus diferentes modalidades asoma como un procedimiento muy sensible a las modificaciones del volumen ventricular, pudiendo detectar alteraciones en la función miocárdica antes de que la FE se vuelva patológica.

CONCLUSIONES

El cierre percutáneo del CAP frecuentemente provoca una significativa y temprana disfunción ventricular izquierda que en la mayoría de los casos se recupera espontáneamente pocas semanas después. El diámetro del conducto arterioso, el volumen del VI y la deformidad miocárdica antes del cierre podrían utilizarse como predictores de deterioro de la función miocárdica luego del procedimiento. 
AGRADECIMIENTO

Los autores expresamos nuestro agradecimiento al Ing. PHD Marco Buestán B. por su valiosa asistencia en la gestión estadística

CONFLICTO DE INTERESES:

Los autores declaran que el presente trabajo de investigación es original, no ha sido publicado con anterioridad ni ha sido ofrecido a ningún otro medio de publicación.

En la elaboración del presente trabajo los autores declaramos estar libres de conflictos de interes llámense laborales, económicos o morales.

\section{REFERENCIAS BIBLIOGRÁFICAS}

1. Schneider DJ, Moore JW Patent Ductus Arteriosus Circulation 2006;114:1873-1882 disponible en http://www.circulationaha.org doi: 10.1161/CIRCULATIONAHA.105.5920631873.

2. Ramaciotti C, Lemler MS, Moake L, RN, Zellers TM Comprehensive Assessment of Patent Ductus Arteriosus by Echocardiography Before Transcatheter Closure J Am Soc Echocardiogr 2002; 15:1154-9.

3. Bilkis AA, Alwi M, Hasri S, Haifa AL, Geetha K, Rehman MA, Hasanah I The Amplatzer Duct Occluder: Experience in 209 Patients J Am Coll Cardiol 2001;37:258-61.

4. Jeong Y-H, Yun T-J, Song J-M, Park J-J, Seo D-M, Koh J-K, Lee S-W, Kim M-J, Kang D-H, Song J-K Left ventricular remodeling and change of systolic function after closure of patent ductus arteriosus in adults: Device and surgical closure Am Heart J 2007; 154:436240 doi:10.1016/j.ahj.2007.04.045

5. Eerola A, Jokinen E, Boldt T, Pihkala J The Influence of Percutaneous closure of Patent Ductus Arteriosus on Left Ventricular Size and Function A Prospective Study Using Two- and Three-Dimensional Echocardiography and Measurements of Serum Natriuretic PeptidesJ Am Coll Cardiol 2006; 47(5):1060-1066 disponible en https://content.onlinejacc.org/ doi:10.1016/j.jacc.2005.09.067

6. Durán M, Freire I Estudio de la función miocárdica mediante ecocardiografía con speckle tracking. Valores normales en un población pediátrica Medicina 2015; 19 (1) 10-20.

7. Elsheikh RG, Hegab MS, Salama MM, Elseteha ME, Omar A Echocardiograpic evaluation of shortterm outcome of Patent Ductus Arteriosus closure using Amplatzer Occluder Device J Cardiovasc Dis Diagn 2015;3:5 doi.org/10.4172/2329-9517.1000220
8. Kim YH, Choi HJ, Cho Y, Lee SB, Hyun MC Transient left ventricular dysfunction after percutaneous Patent Ductus Arteriosus closure in children Korean Circ J 2008; 38:596-600 doi 10.4070 / kcj.2008.38.11.596.

9. Galal MO, Amin M, Hussein A, Kouatli A, Al-Ata J, Jamjoom A Left Ventricular Dysfunction after Closure of Large Patent Ductus Arteriosus Asian Cardiovasc Thorac Ann 2005;13:123-126.

10. Takahashi Y, Harada K, Ishida A, Tamura M, Tanaka T, Takada G Changes in left ventricular volume and systolic function before and after the closure of ductus arteriosus in full-term infants Early Human Development 1996; 44:77-85.

11. Gupta SK, Krishnamoorthy KM, Tharakan JA, Sivasankaran S, Sanjay G, Bijulal S, Anees T Percutaneous closure of patent ductus arteriosus in children: Immediate and short-term changes in left ventricular systolic and diastolic function Ann Pediatr Cardiol. 2011; 4(2): 139-144. doi: 10.4103/0974-2069.84652

12. Burns AT, La Gerche A, D'hooge J, MacIsaac AI, Prior DL Left ventricular strain and strain rate: characterization of the effect of load in human subjects Eur J Echocardiogr 2010; 11: 283-289 doi:10.1093/ejechocard/jep214

13. EL-Khuffash AF, Jain A, Weisz D, Mertens L, McNamara PJ Acute Changes in Myocardial Systolic Function in Preterm Infants Undergoing Patent Ductus Arteriosus Ligation: A Tissue Doppler and Myocardial Deformation Study J Am Soc Echocardiogr 2012;25:1058-67 disponible en http://dx.doi. org/10.1016/j.echo.2012.07.016

14. Agha HM, Hamza HS, Kotby A, Ganzoury M E L, Soliman Nanies Predictors of transient left ventricular dysfunction following transcatheter patent ductus arteriosus closure in pediatric age J Saudi Heart Assoc (2017).

15. Ghonimy R, Adel M, Hegab M, Abdelaziz O, Khairee $\mathrm{E}$ Strain rate imaging for the evaluation of left ventricular function after Patent Ductus Arteriosus closure Heart Mirror J 2011; 5(3): 387-392) disponible en http:www.heartmirror.com 